\title{
Identification of Strict Left Bundle Branch Block, Using a Moving Dipole Model
}

\author{
Werner Bystricky \\ TSD, Langenburg, Germany
}

\begin{abstract}
This study did investigate how well a moving dipole approximation of the electrical activity of the heart can contribute in differentiating strict left bundle branch block (sLBBB) from other, unspecified depolarization abnormalities.

561 ECGs with given $S L B B B$ diagnoses from the $L B B B$ initiative of the ISCE 2018 meeting were used for applying a moving dipole model to the individual heart beats, providing the 3-dimensional time courses of the dipole's position and momentum. The dipole position and momentum traces plus sex and QRS width were used as input for a logistic regression model. The classification performances of the various input dataset were compared by calculating the area under the receiver operating characteristic curve (AUC) and through a two-fold cross validation approach.

Best classification performance was observed using 10 ms time segments including 9 dipole position and 12 dipole momentum parameters with average cross validation AUC value of 0.920 (95\% CI [0.896; 0.945]).

The moving dipole analysis based on the standard 12lead ECG provides new and physiologically relevant information about the location of the electrical activity during depolarization, which may help in improving the assessment of depolarization abnormalities.
\end{abstract}

\section{Introduction}

2011, Strauss et al [1] proposed stricter criteria for complete left bundle branch block diagnosis with the purpose to identify subjects that mostly benefit for cardiac resynchronization therapy (CRT). Besides longer QRS duration ( $\geq 140 \mathrm{~ms}$ for men and $\geq 130 \mathrm{~ms}$ for women) and QS- or rS-configuration in the leads V1 and V2, midQRS notching or slurring in at least 2 contiguous leads was requested to meet SLBBB criteria. Visual assessment of the sLBBB criteria using the standard 12-lead ECG may be subjective. Thus, automated algorithms for identifying sLBBB may improve CRT.

This study aims in evaluating to which extend SLBBB conditions can be derived from a moving dipole approximation of the electrical activity of the heart, omitting the assessment of specific waveform patterns like notching and slurring.

\section{Data}

This study uses the ECGs that have been published as part of the LBBB initiative of the ISCE 2018 meeting [2]. The ECGs are 10-second excerpts, extracted from 12-lead Holter recordings with Mason-Likar lead configuration, captured before CRT implantation in the MADIT-CRT trial [3]. All ECGs have been adjudicated for strict LBBB presence according to [1]. The ECGs were split in a training dataset $\mathrm{A}$ and in a test dataset $\mathrm{B}$ of about equal size. Each dataset also contained 20 duplicate ECGs for testing method reproducibility. Omitting the duplicate ECGs, the dataset consisted of 561 ECGs with annotations as shown in table 1 .

Table 1. ECG datasets

\begin{tabular}{lccc}
\hline Dataset & sLBBB & Not sLBBB & $\sum$ \\
\hline A (training) & 160 & 120 & 280 \\
B (test) & 142 & 139 & 281 \\
Male & 188 & 215 & 403 \\
Female & 114 & 44 & 158 \\
\hline
\end{tabular}

\section{Moving Dipole Analysis}

The moving dipole model describes the electrical activity of the heart in terms of a single, time varying dipole with momentum $q(t)$ at position $p(t)$. The electrical activity is measured at the body surface by the 8 leads I, II, V1, to V6. At every time instance $t_{i}$, a measurement $m_{L}\left(t_{i}\right)$ at lead $\mathrm{L}$ may be considered as scalar product of the dipole momentum with the appropriate lead vector. The lead vector for a given pair of electrodes depends on the electrode positions, the time varying position of the dipole itself, as well as on properties of the volume conductor (body geometry, tissue conductivities). For an infinite, homogeneous volume conductor with conductivity $\sigma$ the potential $\Phi$ of 
a dipole diminishes with distance $r$ from its position as $\Phi(\mathrm{r}) \cong 1 /_{\sigma r^{2}}($ see $[4])$.

For a pair of electrodes $E_{j}$ and $E_{k}$ at position $e_{j}$ and $e_{k}$ measuring the potential difference $\Phi\left(e_{j}\right)-\Phi\left(e_{k}\right)$, the corresponding lead vector $l_{j, k}(t)$ is:

$$
l_{j, k}(t)=\frac{e_{j}-p(t)}{\sigma\left\|e_{j}-p(t)\right\|^{3}}-\frac{e_{k}-p(t)}{\sigma\left\|e_{k}-p(t)\right\|^{3}}
$$

For an inhomogeneous and finite thorax, formula (1) may be adjusted by allowing different conductivities related to the individual electrodes and by (slight) deviation of the effective electrode positions from the physical positions. A lead measurement $m_{j, k}\left(t_{i}\right)$ may be predicted as:

$$
\widehat{m}_{j, k}\left(t_{i}\right)=l_{j, k}\left(t_{i}\right) \cdot q\left(t_{i}\right)
$$

In this study, the equivalent moving dipole generator was estimated by minimizing the expression:

$$
\begin{aligned}
\sum_{i=1}^{N}\left\|m\left(t_{i}\right)-\widehat{m}\left(t_{i}\right)\right\|^{2}+\Phi_{p}(p)+\Phi_{q}(q) \\
+\Phi_{e}(e, \tilde{e})+\Phi_{\sigma}(\sigma, \tilde{\sigma}) \rightarrow \min
\end{aligned}
$$

where $N$ is the number of sampling points, $m\left(t_{i}\right)$ and $\widehat{m}\left(t_{i}\right)$ the measurement vector respectively the prediction vector of all leads at time instance $t_{i} . \Phi_{\chi}(\chi, \tilde{\chi})$ denotes some regularizer function related to the model parameter $\chi$ and its optional prior value $\tilde{\chi}$. Further details are given in [5].

For each ECG, the moving dipole model was fitted to the heart beats lying in the central 5 seconds, ending up in 2403 analyzed beats. For each analyzed beat, the average moving dipole position and momentum within the QRS complexes were calculated in time slices of length 160 , $80,40,20$, and $10 \mathrm{~ms}$ between the QRS onset and the next $160 \mathrm{~ms}$. The spatial $\mathrm{X}, \mathrm{Y}$, and $\mathrm{Z}$ components of these averages were used as input for the following classification approach, as well as the QRS width, which was determined per beat by a proprietary ECG analysis system.

\section{Classification}

To differentiate between sLBBB and non-sLBBB, a logistic regression approach [6] was applied using various subsets of the position and momentum data from the time slices, plus sex and the QRS width. Significant classification parameters were identified using a genetic algorithm by optimizing the Bayesian information criterion. The classification performance for the various input subsets was compared through the area under receiver operating characteristic (AUC), as well as through a 2-fold cross validation approach with 1000 randomly chosen ECG subsets, mirroring the conditions of the learning/test datasets in the ISCE LBBB challenge.

\section{Results}

Figure 1 displays the performances of various input subsets for separating sLBBB from non-sLBBB conditions. Best separation was observed for M6, consisting of the input parameter sex, QRS width, as well as 9 position and 12 momentum parameters from the $10 \mathrm{~ms}$ time slices. Fitting M6 to the training dataset and predicting $\mathrm{SLBBB}$ on the test dataset resulted in the performance measures displayed in table 2. Sensitivity, specificity, and accuracy were all in the order of 0.85 .

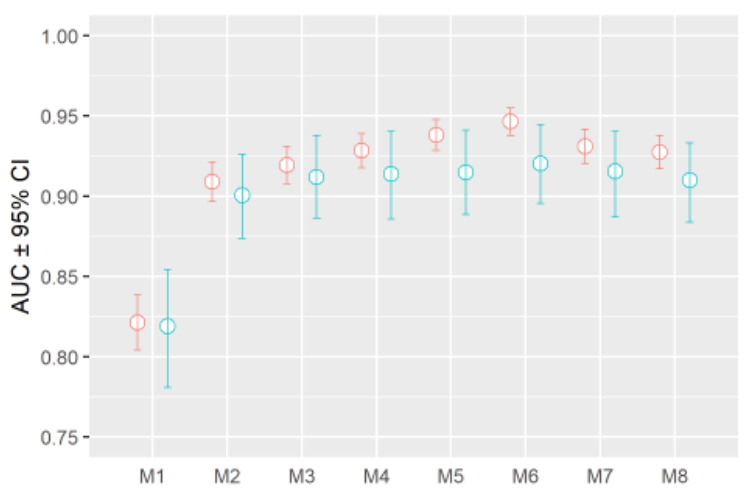

Figure 1. Classification performance for 8 different input models, each of them using sex and QRS width as input parameter. M1: Just sex + QRS width; M2: 160ms slices; M3: 80ms time slices; M4: 40ms time slices; M5: 20ms time slices; M6: 10ms time slices; M7: 10ms time slices, momentum only; M8: 10ms time slices, position only. Red: Fit to all data; Blue: Cross validation.

Table 2. Contingency table for model M6, fit to training dataset and prediction on test dataset.

\begin{tabular}{llccc}
\hline & & \multicolumn{3}{c}{ Annotation } \\
& & sLBBB & Not sLBBB \\
\hline \multirow{2}{*}{ Prediction } & sLBBB & 122 & 22 & 0.847 \\
& Not sLBBB & 20 & 117 & 0.854 \\
& & 0.859 & 0.842 & 0.851 \\
\hline
\end{tabular}

Figure 2 provides details about the classification performance of M6, showing an AUC value of 0.946 for fitting the M6 to all data with $95 \%$ confidence interval [0.938; 0.955]. Cross validation of M6 resulted in an average AUC value of 0.920 with $95 \%$ confidence interval [0.896; 0.945].

As depicted in figure 1, adding moving dipole signal data to sex and QRS width increased the classification performance from AUC $=0.82$ to values larger than 0.90 . Smaller time slices performed better than larger time slices. Using the $10 \mathrm{~ms}$ time slices with momentum data only (model M7) performed slightly better than using position data only (model M8). The significant moving dipole parameters are depicted in figure 3 . 


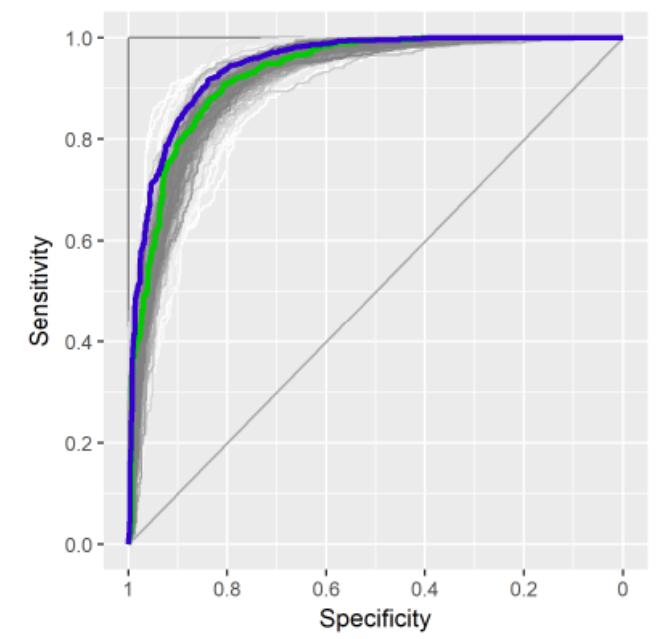

Figure 2. Classification performance for model M6. Blue: Fit to all data. Green: Fit to training data, prediction on test data. Grey: Cross validation.

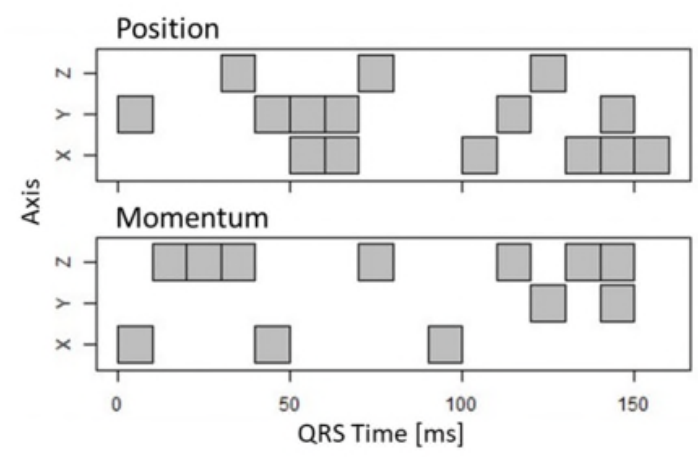

Figure 3. Significant moving dipole parameters using $10 \mathrm{~ms}$ time slices. Top: Model M8, position data only. Bottom: Model M7, momentum data only.

\section{Discussion}

The classification described here was based on the given sLBBB annotations, which depend on the QRS duration measurement, the visual determination of QS- or rS-configurations in leads V1 and V2, and on the visual determination of mid-QRS notching or slurring. This sLBBB assessment might have been affected by subjective decisions, and may not absolutely reflect true physiological LBBB conditions.

QS- and rS-configurations, as well as notching conditions were not explicitly modelled here. The moving dipole description of the QRS signal encodes these characteristics to some extent, as indicated by the classification performance gain when adding the moving dipole data to sex and the QRS width.
The moving dipole's position trajectory is new information that cannot be visually deducted from the standard 12-lead ECG. Notably, this position information improves sLBBB classification in addition to the pure momentum information (compare models M8, M7, and M6 in figure 1).

Under complete LBBB, depolarization starts in the right ventricle, passes the ventricular septum, and propagates through the left ventricle [1]. Thus, depolarization under LBBB is mainly characterized by its location trajectory. The classical LBBB criteria, as well as the strict LBBB criteria, derive location information indirectly from the heart's dipole momentum vector. The moving dipole model presented here, however, produces explicit information about the excitation location trajectory as depicted in figure 4: The panels A and B display $140 \mathrm{~ms}$ long moving dipole trajectories of the ventricular depolarization from two male subjects, both with 150ms broad QRS complexes and rS configuration in V1 and V2. In panel A (displaying an ECG with sLBBB annotation), the center of excitation immediately moves to the body's right and front side, and then progresses to the left side. This may be considered as the expected excitation process for complete LBBB. In panel $B$, the center of excitation starts moving to the body's left and front side, turns back to the heart's center, and proceeds with a turn to the very left and front side, finally returning to the center of the heart. This ECG was not annotated as sLBBB because only one notch was identified in lead I while all other SLBBB criteria were fulfilled. However, the moving dipole estimate unveils more pronounced heterogeneities in the trajectories of the dipole position and the dipole momentum compared to A, what may better reflect excitation conditions than pure standard 12-lead ECG based waveform properties.

Of note: One might expect that the path of the moving dipole's position should be in line with the direction of the dipole vector. The moving dipole approximation shows that this is usually not the case. Moreover, the dipole momentum typically is rather orthogonal to the moving dipole's position trajectory. This observation was also reported by Vito Starc (personal communication, CinC 2018) who applied a similar moving dipole approach to healthy subjects [7]. A reason for this misalignment may relate to the fact that the moving dipole's position and momentum are calculated as a spatial integral over the whole excitation wave front, while the excitation propagation on the cellular level may be determined by the local dipole orientation and by myocardial structures. 


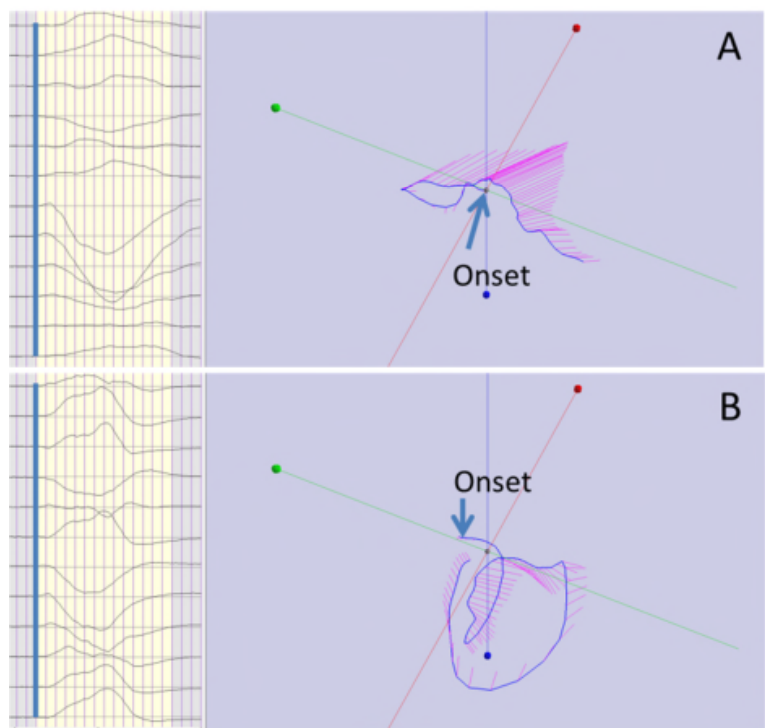

Figure 4. Moving dipole of single QRS complexes.

A: sLBBB; B: Non-sLBBB. Left: 12-lead ECG. Right: Moving dipole at QRS onset plus $140 \mathrm{~ms}$. Blue lines: Dipole position. Red lines: Dipole momentum. Coordinate needles: Red: Back to front. Green: Right to left. Blue: Foot to head.

The moving dipole analysis did identify the expected shift of the early excitation path towards the right ventricle under LBBB for the entire population as shown in figure 5.

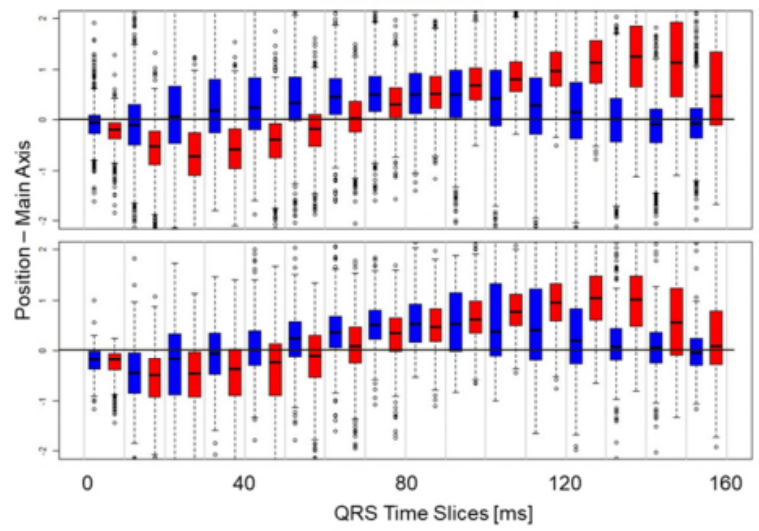

Figure 5. Distribution of the moving dipole position within the QRS complex along the main axis (see text).

Top: male. Bottom: female.

Red: sLBBB. Blue: non-sLBBB.

The largest difference of the dipole location between sLBBB and non-sLBBB was observed when viewing along a main axis pointing from the body's right, front, top side to left, back, bottom. The average dipole position between 10 and $60 \mathrm{~ms}$ after $\mathrm{Q}$ was more negative for sLBBB (red bars) compared to non-sLBBB (blue bars), and clearly more positive at the end of depolarization. This difference in the moving dipole's position between sLBBB and non-sLBBB was less pronounced for female than for male subjects.

\section{Conclusion}

sLBBB ECGs could be separated from non-sLBBB ECGs with an accuracy of $85 \%$. Significant parameters for sLBBB classification were sex, QRS width, and components from both, position and momentum, of the moving dipole.

The moving dipole analysis provides physiologically relevant information about the excitation process that is not available by standard ECG assessment. Most notably, the moving dipole analysis did unveil a systematic shift of the early excitation path under sLBBB conditions to the body's front and right side as expected for a delayed excitation of the left ventricle.

The moving dipole analysis may contribute to an enhanced assessment of depolarization abnormalities even beyond LBBB.

\section{References}

[1] Strauss DG, Selvester RH, Wagner GS. Defining Left Bundle Branch Block in the Era of Cardiac Resynchronization Therapy. The American Journal of Cardiology 2011;107: 927 - 934.

[2] LBBB Initiative of the ISCE 2018 meeting. http://www.thew-project.org/LBBB_Initiative.htm

[3] Moss AJ et al. Cardiac-Resynchronization Therapy for the Prevention of Heart-Failure Events. New England Journal of Medicine 2009;361:1329-1338.

[4] Malmivuo J. Biomagnetism. In Wiley Encyclopedia of Electrical and Electronics Engineering. Webster JG (Ed) 1999

[5] Bystricky W. Modelling the Electrical Activity of the Heart by a Single Dipole, Concurrently Estimating Subject and Measurement Related Conditions. US 8,290,576 B2. 2012.

[6] R Core Team. R: A Language and Environment for Statistical Computing. Vienna, Austria; 2017. https://www.R-project.org/R System

[7] Starc V, Swenne C. Spatial Distribution and Orientation of a Single Moving Dipole Computed in 12-Lead ECGs of a Healthy Population Using a Spherically Bounded Model. Computing in Cardiology 2017;44:242-277.

Address for correspondence.

Werner Bystricky

Am Kelterplatz 8

74595 Langenburg

Germany

tsd@werner-bystricky.de 